\title{
Gut Microbiota and Depressive Symptoms at the End of CRT for Rectal Cancer: A Cross-Sectional Pilot Study
}

\author{
Velda J. Gonzalez-Mercado ${ }^{\mathrm{D}},{ }^{1}$ Jean Lim, ${ }^{2}$ Leorey N. Saligan, ${ }^{3}$ Nicole Perez, ${ }^{1}$ \\ Carmen Rodriguez, ${ }^{4}$ Raul Bernabe, ${ }^{5}$ Samia Ozorio, ${ }^{4}$ Elsa Pedro, ${ }^{6}$ Farrah Sepehri, ${ }^{4}$ \\ and Brad Aouizerat ${ }^{7}$ \\ ${ }^{1}$ Rory Meyers College of Nursing, New York University, New York, NY, USA \\ ${ }^{2}$ University of Miami, Miami, FL, USA \\ ${ }^{3}$ Intramural Program, National Institute of Nursing Research/National Institute of Health, Bethesda, MD, USA \\ ${ }^{4}$ College of Nursing, University of South Florida, Tampa, FL, USA \\ ${ }^{5}$ Department of General Studies, University of Puerto Rico, San Juan, Puerto Rico \\ ${ }^{6}$ School of Pharmacy, Medical Science Campus, University of Puerto Rico, San Juan, Puerto Rico \\ ${ }^{7}$ Bluestone Center for Clinical Research, Department of Oral and Maxillofacial Surgery College of Dentistry, New York University, \\ New York, NY, USA
}

Correspondence should be addressed to Velda J. Gonzalez-Mercado; vg2145@nyu.edu

Received 25 May 2021; Accepted 24 November 2021; Published 29 December 2021

Academic Editor: Marcin Rzeszutek

Copyright (c) 2021 Velda J. Gonzalez-Mercado et al. This is an open access article distributed under the Creative Commons Attribution License, which permits unrestricted use, distribution, and reproduction in any medium, provided the original work is properly cited.

\begin{abstract}
Background. The role of alterations in gut microbiota composition (termed dysbiosis) has been implicated in the pathobiology of depressive symptoms; however, evidence remains limited. This cross-sectional pilot study is aimed at exploring whether depressive symptom scores changed during neoadjuvant chemotherapy and radiation therapy to treat rectal cancer, and if gut microbial taxa abundances and predicted functional pathways correlate with depressive symptoms at the end of chemotherapy and radiation therapy. Methods. 40 newly diagnosed rectal cancer patients (ages 28-81; 23 males) were assessed for depressive symptoms using the Hamilton Rating Scale for Depression (HAM-D) and provided stool samples for 16S rRNA sequencing. Gut microbiome data were analyzed using QIIME2, and correlations and regression analyses were performed in R. Results. Participants had significantly higher depressive symptoms at the end as compared to before CRT. The relative abundances of Gemella, Bacillales Family XI, Actinomyces, Streptococcus, Lactococcus, Weissella, and Leuconostocaceae were positively correlated (Spearman's rho $=0.42$ to 0.32), while Coprobacter, Intestinibacter, Intestimonas, Lachnospiraceae, Phascolarctobacterium, Ruminiclostridium, Ruminococcaceae (UCG-005 and uncultured), Tyzzerella, and Parasutterella (Spearman's rho $=-0.43$ to -0.31 ) were negatively correlated with HAM-D scores. Of the 14 predicted MetaCyc pathways that correlated with depressive symptom scores at the end of CRT, 11 (79\%) were associated with biosynthetic pathways. Conclusions. Significant bacterial taxa and predicted functional pathways correlated with depressive symptoms at the end of chemotherapy and radiation therapy for rectal cancer which warrants further examination and replication of our findings.
\end{abstract}

\section{Introduction}

Rectal cancer is prevalent worldwide, with more than 43,000 new patients in the US annually [1]. A large proportion of newly diagnosed rectal cancer patients undergo preoperative neoadjuvant chemotherapy and radiation therapy (CRT) $[2$, 3]. In addition to the physiological side effects (e.g., diarrhea and constipation), cancer and/or CRT can negatively influence patients' psychological health, precipitating mood disorders, including depression $[4,5]$. The prevalence of depression during active treatment (e.g., patients surgically treated) for rectal cancer may be as high as $47 \%$ [6], and depression continues to affect $24-31 \%$ of patients after treatment completion [4]. However, the literature on the 
prevalence of depressive symptoms and whether depressive symptom scores changed during CRT for rectal cancer is not available.

Depressive symptoms frequently include loss of interest, sadness, irritability, feelings of worthlessness, hopelessness, guilt or anxiety, concerns over death, or suicidal ideation, as well as associated symptoms such as changes in appetite, weight loss or weight gain, sleep disturbances, psychomotor activity, decreased energy, indecisiveness, or distracted attention [7]. Further, depressive symptoms in cancer patients have been reported to be associated with poor quality of life and lower survival $[6,8]$. Unfortunately, depression in patients often goes undiagnosed and undertreated in part because of the lack of laboratory-based diagnostic and prognostic tools [9]. Evidence suggests that the perturbation of the gut microbiota (defined as a diverse collection of microorganisms that inhabit the gastrointestinal tract; [10]) can influence depressive symptoms via the gut-brain axis and that depression phenotyping focusing on the gut microbiome may produce a microbiota-based signature that can serve as a biomarker for depression $[11,12]$.

Changes in gut microbiota composition induced by factors such as cancer or its treatment have been suggested to be associated or potentially causal of dysbiosis (a disruption in the balance, diversity, and function of symbiotic intestinal microbial communities) $[2,13]$ in depression and other comorbidities/symptoms [2, 11, 14-16]. We recently reported reduced alpha diversity (Shannon Diversity and Observed Operational Taxonomic Units (OTUs)) in rectal cancer patients in the middle and at the end of CRT compared to before CRT, suggesting that CRT is associated with alterations in gut microbiota composition [15]. However, few microbiome studies had identified specific roles of bacterial taxa in cancer-related comorbidities such as depression. Therefore, this pilot study is aimed at exploring whether depressive symptom scores changed during CRT, and if gut microbial taxa abundances and predicted functional pathways correlate with depressive symptoms at the end of CRT in rectal cancer participants who were not taking antidepressants. Results of this study will further our understanding of the underpinnings of CRT-related depressive symptoms and may inform future strategies for the development of microbial signature/biomarkers to detect intensification of depressive symptoms placing patients at risk for acute depression during CRT in rectal cancer patients.

\section{Methods}

2.1. Study Sample and Setting. Prior to data collection, the study protocol received approval by the Institutional Review Board from the Southeastern Academic Medical Center. Patients interested in participating in this study were screened by the research team for eligibility using a brief health history interview that included relevant questions about their cancer history, cancer treatments, current medications, and other health conditions. Forty newly diagnosed rectal cancer patients programmed to receive CRT were included in this study. Eligibility criteria have been described in our previous publications including the following: (1) being at least 18 years of age; (2) no history of inflammatory conditions, infectious conditions, other cancers, sleep disorders, or comorbidities associated with sleep disturbance (e.g., sleep apnea); and (3) with no prior use of antidepressant medications, anti-insomnia medications, antibiotics, probiotics, prebiotics, steroids, or immune-suppressant agents one month before each assessment time-point $[2,14,15,17,18]$. Data collection was conducted from September 2017 to April 2019 at three ambulatory radiotherapy facilities in Tampa Bay, Florida, United States. All procedures performed in our study were in accordance both with the ethical standards of the institutional and/or national research committees and with the 1964 Helsinki Declaration and its later amendments or comparable ethical standards. Written informed consents were provided by all participants.

\subsection{Demographic Characteristics, Clinical Information, and} Assessment of Depressive Symptoms. After obtaining informed consent, study participants completed the demographic questionnaire including information on age, gender, and occupation. The research team recorded information from the medical chart including the following: tumor stage, type of chemotherapy, number of prescribed RT fractions, before CRT hemoglobin ( $\mathrm{Hgb}$ ) levels, and body mass index (BMI). Information for each variable is summarized in Table 1. Depression was assessed by the clinician-rated 17item Hamilton Rating Scale for Depression (HAM-D ${ }_{17}$ ) [19], using the conventional Structured Interview Guide for the HAM-D $[17,20]$. The forms were administered prior, and at the last week of CRT treatment. Hamilton [19] provides detailed information on the HAM-D ${ }_{17}$ scale. Overall acceptable psychometric properties have been reported for the HAM-D, including internal consistency [21]. In our study, the Cronbach alphas for the HAM-D ${ }_{17}$ was 0.86 at before treatment and 0.84 at the end of CRT. A cutoff score of $\geq 15$ has been used previously in cancer patients to screen for depression, with higher scores indicating more symptoms of depression $[17,22]$.

Total DNA was isolated from $250 \mathrm{mg}$ stool aliquots collected at the end of CRT using the PowerSoil DNA Isolation Kit (MoBio, Carlsbad, CA), per the manufacturer's protocol. The V3-V4 regions of the 16S rRNA gene were amplified and sequenced using Illumina's 16S rRNA gene library preparation and sequencing workflow. Reads were trimmed at a $Q=25$ threshold using Trim Galore! v0.44, denoised into amplicon sequence variants (ASVs) using the DADA2 plugin in QIIME2-2019.17, and the resulting ASVs were taxonomically assigned against the SILVA v132 database using a naïve Bayes classifier [16]. The ASV table was rarefied to 4,226 sequences per sample and used for alpha and beta diversity calculations in QIIME2. MetaCyc [23] pathway abundances were predicted from the rarefied table using QIIME2's q2-picrust2 plugin [24].

2.3. Statistical Analyses. Stool sample collection and $16 \mathrm{~S}$ rRNA gene analysis procedures have been described previously by our group [2, 17]. Statistical differences between depression scores were computed using a two-tailed $t$-test with a significance threshold of 0.05 . Associations between 
TABLE 1: Summary of measurements.

\begin{tabular}{|c|c|c|}
\hline Variables & Method of measurement & Frequency \\
\hline Depressive symptoms & $\begin{array}{l}\text { 17-item Hamilton Rating Scale for } \\
\text { Depression }\left(\text { HAM-D }{ }_{17}\right)\end{array}$ & $\begin{array}{l}\text { Before and end of } \\
\text { treatment }\end{array}$ \\
\hline Gut microbiome & Stool samples for $16 \mathrm{~S}$ rRNA sequencing & End of treatment \\
\hline Age, gender, occupational status & Demographic form & Before treatment \\
\hline $\begin{array}{l}\text { Type of chemotherapy, tumor stage, number of treatments, body } \\
\text { mass index, hemoglobin }\end{array}$ & $\begin{array}{l}\text { Health form information gathered from their } \\
\text { health record }\end{array}$ & Before treatment \\
\hline
\end{tabular}

depressive symptom scores and microbial diversity variables were assessed using the Spearman correlation test on samples collected at the end of CRT. To fit a multiple linear regression model that adjusted for important confounding variables (e.g., age), Spearman correlations were also calculated between depressive symptom scores and taxa and pathway abundances for end of CRT samples. Automatic variable selection was performed on genera and pathways whose abundances were statistically significantly correlated with depressive symptom scores using the regsubsets function in the leap package in $\mathrm{R}$. The subset of variables producing the highest $R^{2}$ was used to build the final permutation-based multiple linear regression model using the lmp function in the lmPerm package in R. A $p$ value cutoff of 0.05 is used for all statistical tests.

\section{Results}

3.1. Patient Characteristics and Depressive Symptom Scores. Study participants $(n=40)$ had a mean age of 58.9 years \pm 11.3, and 58\% were males (Table 2). Participants showed significantly higher mean depressive symptom scores $(12.4 \pm 8.7)$ indicating higher severity of depression at the end than before CRT $(9.4 \pm 7.7, t(47)=-3.1, p<0.003)$. Ten participants (25\%) before CRT and fifteen participants (38\%) at the end of CRT had depressive symptom scores that exceeded the cutoff score for depression of $\geq 15[17,22]$.

3.2. The Gut Microbiome Is Associated with Depressive Symptom Scores at the End of CRT. In this cross-sectional pilot study, we observed no significant associations between alpha diversity, phylum abundances, and depressive symptom scores at the end of CRT (data not shown). At the genus level, however, we found that the relative abundances of Firmicutes genera, including Gemella, Bacillales Family XI, Streptococcus, Lactococcus, Weissella, and Leuconostocaceae, as well as Actinomyces (phylum Actinobacteria), were positively correlated with depressive symptom scores (Spearman's rho $=0.32$ to 0.42 ). On the other hand, negative correlations between depressive symptom scores and the abundances of Coprobacter (phylum Actinobacteria), Parasutterella (phylum Proteobacteria), and other Firmicutes genera Intestinibacter, Intestimonas, Lachnospiraceae NK4A136 group, Phascolarctobacterium, Ruminiclostridium, Ruminococcaceae (UCG-005 and uncultured), and Tyzzerella were observed (Spearman's rho $=-0.43$ to -0.31 ) (Table 3).

From the ASV abundance data, 14 predicted MetaCyc [23] pathways were significantly correlated with depressive symptom scores at the end of CRT (Table 4). Eleven $(\sim 79 \%)$ of these were biosynthetic pathways, of which six (i.e., biosynthesis of inosine- $5^{\prime}$-phosphate, L-methionine (pathways I and III), mono-trans, poly-cis decaprenyl phosphate, L-alanine, and S-adenosyl-L-methionine) were positively correlated with depressive symptoms, while five (i.e., biosynthesis of CMP-legionaminate, flavin, GDP-mannose, phosphopantothenate, and coenzyme A) were negatively correlated with depressive symptom scores (Table 4). The remaining three nonbiosynthetic pathways were negatively correlated with depressive symptom scores and included acetyl-CoA fermentation to butanoate, chondroitin sulfate degradation, and myo-, chiro-, and scyllo-inositol degradation pathways (Table 4). Further, with respect to multiple regression, automatic subset selection bacterial taxa measured at the end of RT and predicted pathway abundance variables showed that Bacillales Family XI and Ruminococcaceae UCG-005 general along with the predicted abundances of coenzyme A biosynthesis and myo-, chiro-, and scyllo-inositol degradation pathways were optimally negatively and positively correlated with depressive symptom scores, after controlling for age (Figure $1 ; R^{2}=$ 0.37 ; adjusted $R^{2}=0.28$; $\mathrm{SE}$ of estimate $=7.16 ; F\left({ }_{5,34}\right)=$ 3.98, $p=0.006)$.

\section{Discussion}

In this pilot study, we aimed to explore whether depressive symptom scores changed during CRT to treat rectal cancer, and if gut microbial taxa abundances and predicted functional pathways correlate with depressive symptoms at the end of CRT. While depressive symptoms are common in the general population worldwide [25], depression in the cancer population is a major health concern because it is often associated with poor adherence to treatment, higher tumor recurrence, and lower survival rates $[6,8,26]$. Our study showed that rectal cancer patients may present with increased levels of depressive symptoms even before treatment. This finding is important given that previous reports have also shown that depression is frequently underdiagnosed and undertreated $[22,27]$. The physical and psychological strain associated with a patient's cancer diagnosis may be interpreted as a normal response to cancer and/or its treatment, and in the absence of a standard of care that supports ongoing clinical assessment for depression, failure to identify symptoms of depression may occur. Reasons for underdiagnosis and undertreatment of depression reported 
TABle 2: Study participants' $(n=40)$ demographic and clinical characteristics.

\begin{tabular}{lc}
\hline Characteristics & Participants $(n=40)$ \\
\hline Gender & $23(58 \%)$ \\
$\quad$ M & \\
Occupation & $17(42 \%)$ \\
$\quad$ Working & $13(33 \%)$ \\
Retired & $10(25 \%)$ \\
Handicapped & \\
Chemotherapy & $17(42 \%)$ \\
5FU & $23(58 \%)$ \\
$\quad$ Xeloda & Median $(\mathrm{IQR})$ \\
& $58(53,67)$ \\
Age & $3(3,3)$ \\
Tumor stage & $28(23,33)$ \\
Number of treatments & $27.5(23.6,30.1)$ \\
BMI & $11.8(10.7,13.2)$ \\
Hemoglobin &
\end{tabular}

$\mathrm{BMI}=$ body mass index.

TABLE 3: Statistically significant correlations between genus relative abundances and the Hamilton Depression Rating Scale scores at the end of CRT.

\begin{tabular}{|c|c|c|}
\hline \multirow{2}{*}{ Genus } & \multicolumn{2}{|c|}{ End-CRT } \\
\hline & Rho & $p$ value \\
\hline \multicolumn{3}{|l|}{ Phylum: Actinobacteria } \\
\hline Actinomyces & 0.40 & 0.01 \\
\hline Actinomycetaceae & 0.40 & 0.01 \\
\hline Actinomycetales & 0.38 & 0.02 \\
\hline \multicolumn{3}{|l|}{ Phylum: Bacteroidetes } \\
\hline Coprobacter & -0.32 & 0.05 \\
\hline \multicolumn{3}{|l|}{ Phylum: Firmicutes } \\
\hline Bacillales Family XI & 0.42 & 0.007 \\
\hline Gemella & 0.42 & 0.007 \\
\hline Intestinibacter & -0.37 & 0.02 \\
\hline Intestinimonas & -0.43 & 0.005 \\
\hline Lachnospiraceae NK4A136 group & -0.32 & 0.05 \\
\hline Lactococcus & 0.34 & 0.03 \\
\hline Phascolarctobacterium & -0.32 & 0.04 \\
\hline Ruminiclostridium 6 & -0.39 & 0.01 \\
\hline Ruminococcaceae UCG-005 & -0.32 & 0.05 \\
\hline Ruminococcaceae uncultured & -0.41 & 0.009 \\
\hline Streptococcaceae & 0.37 & 0.02 \\
\hline Streptococcus & 0.37 & 0.02 \\
\hline Tyzzerella & -0.35 & 0.03 \\
\hline Weissella & 0.32 & 0.04 \\
\hline \multicolumn{3}{|l|}{ Phylum: Proteobacteria } \\
\hline Parasutterella & -0.31 & 0.05 \\
\hline
\end{tabular}

$\mathrm{CRT}=$ chemotherapy and radiation therapy. in the literature include treatment-related side effects that can mask depressive symptoms (e.g., fatigue and lack of energy), people with cancer that are expected to have poor mood, reduced time available in the treatment setting, scarce data on depression drug trials among cancer patients, and the lack of biomarkers for depression [9, 22, 27, 28]. Our findings have several clinical implications including the importance of the need for routine depression surveillance, referrals to mental health professionals, treatment of depression, and the importance of patient education before CRT initiation $[2,29]$, as well as the need for non-invasive objective indicator of potential depression.

Although the causal interplay between cancer, CRT, gut microbial composition, and depression remain undercharacterized, emerging evidence suggests that cancer treatments mediate changes in gut microbial composition, causing abnormalities in the gut-brain axis that may result in depressive symptoms. Briefly, one hypothesis is that changes in gut microbial composition can be affected through CRTinduced cellular responses that increase intestinal permeability and facilitate translocation of bacteria associated with peripheral inflammatory immune response [30, 31]. This increases systemic levels of proinflammatory cytokines, leading to behavioral comorbidities such as depressive symptoms [32-34]. More detailed information on the effects of cancer treatments on the gut microbiome [30,31] and its relationship with behavioral comorbidities can be found in recent reviews [32-34]. Because the gut and central nervous systems are linked via a bidirectional communication network [35], another possibility is that the central nervous system sends signals to the gut environment, resulting in modulation of the gut microbiota composition and function [36]. For example, animal studies demonstrated that germfree mice receiving fecal microbiota transplantation from depressed human patients presented more depressive symptoms, and their microbiota were different compared to mice receiving fecal microbiota transplantation from healthy recipients, suggesting that depressive-like symptoms can be transferred via the microbiota [37]. Conversely, probiotic intervention can alleviate and improve depressive symptoms $[38,39]$. On the other hand, cancer-related symptoms, such as muscle wasting (sarcopenia; [40]), body weight loss, weakness, muscle atrophy and fat depletion (cachexia; [41-43]), anorexia [44], and social isolation [45], can also lead to depressive symptoms. In this regard, sarcopenia, cachexia, anorexia, and social isolation are also associated with microbial dysbiosis in preclinical models [41, 46-48]. For example, Pötgens et al. [41] observed an increased abundance of Enterobacteriaceae and altered gut barrier function in animal models of cancer cachexia. Taken together, the relationship between cancer, CRT, gut microbial composition, and depression is potentially complex and warrants further large-scale, controlled studies to elucidate the effects they exert on each other.

We found preliminary associations between abundances of Firmicutes, Actinobacteria, and Proteobacteria taxa and predicted pathways with depressive symptom scores at the end of CRT for rectal cancer. Firmicutes, Actinobacteria, and Proteobacteria are generally dominant in the healthy 
TABLE 4: Statistically significant correlations between MetaCyc pathway abundances and the Hamilton Depression Rating Scale scores at the end-CRT samples.

\begin{tabular}{lcc}
\hline MetaCyc pathway & Rho & End-CRT \\
\hline Acetyl-CoA fermentation to butanoate II & -0.37 & 0.02 \\
Chondroitin sulfate degradation I (bacterial) & -0.33 & 0.04 \\
CMP-legionaminate biosynthesis I & -0.41 & 0.009 \\
Flavin biosynthesis I (bacteria and plants) & -0.36 & 0.02 \\
Inosine-5' -phosphate biosynthesis III & 0.33 & 0.04 \\
GDP-mannose biosynthesis & -0.33 & 0.04 \\
L-methionine biosynthesis I & 0.36 & 0.02 \\
L-methionine biosynthesis III & 0.42 & 0.36 \\
Mono-trans, poly-cis decaprenyl phosphate biosynthesis & -0.39 & 0.007 \\
Myo-, chiro-, and scyllo-inositol degradation & -0.39 & 0.02 \\
Phosphopantothenate biosynthesis I & -0.42 & 0.01 \\
Superpathway of coenzyme A biosynthesis I (bacteria) & 0.36 & 0.02 \\
Superpathway of L-alanine biosynthesis & 0.33 & 0.007 \\
Superpathway of S-adenosyl-L-methionine biosynthesis & & 0.02 \\
\hline
\end{tabular}

$\mathrm{CRT}=$ chemotherapy and radiation therapy.

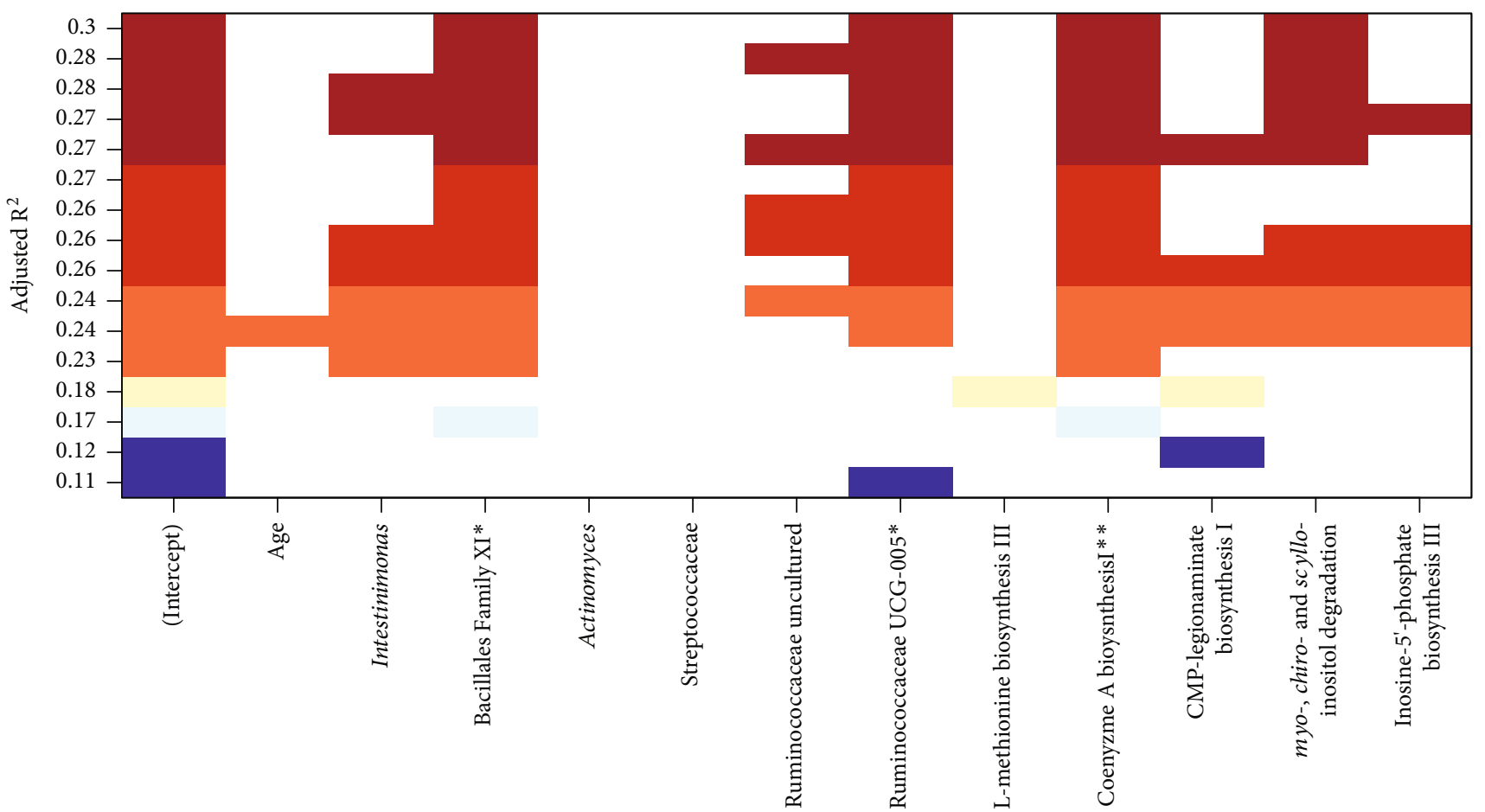

FIGURE 1: All subset regression results showing, for each subset size, the two subsets of predictor variables yielding the highest adjusted $R^{2}$ for predicting depressive symptom scores. * indicates predictors (coefficients) significantly correlated with depression scores, based on permutation test $p$ values in the final multiple regression model.

human gut [49]. However, consistent with our results, several genera from these phyla, including Actinomyces (phylum Actinobacteria), Streptococcus, and Weissella (phylum Firmicutes), have been associated with depression [11, 37, 50-52]. Particularly, higher Streptococcus abundances were detected in patients with major depressive disorders (MDD) using a similar 16S rRNA gene sequencing protocol and the same measure of depression used in our study [52].
Streptococcal infections are linked to depression-like behavior and/or to behavioral abnormalities (i.e., bacterialinfection-induced autoantibodies in pediatric autoimmune neuropsychiatric disorders associated with streptococcal infection (PANDAS)) in animal models $[53,54]$. Polymicrobial infections between Actinomyces species and members of the Streptococcus genus have also been reported [55-57]. Besides infections, associations between Actinomyces strains 
and chronic inflammatory diseases such as Crohn's disease $[58,59]$ and higher depressive symptoms $[60,61]$ have also been reported. Adding to the complex association between gut microbiome and depression, there is also evidence that antimicrobials used to treat depression can result in gut dysbiosis through antimicrobial effects (Ting-Ting $[62,63]$ ). Another Firmicutes genus that was significantly associated with depressive symptom scores, Weissella, was previously identified in infants born to mothers exposed to psychological stressors and distress such as intimate partner violence [64]. Nevertheless, the source of these depression-associated genera in the gut and the exact mechanisms of how they affect or are affected by brain functions and behavior require further study.

Most of the bacteria genera that significantly correlated with depressive symptoms in this study were classified to the phylum Firmicutes. Interestingly, some taxa belonging to the phylum Firmicutes (i.e., Lachnospiraceae, Ruminiclostridium, and Ruminococcaceae) that play roles in the shortchain fatty acid (SCFA) production were inversely associated with depressive symptoms. SCFAs (e.g., acetate, propionate, and butyrate) are microbially derived metabolites produced by the bacterial fermentation of dietary carbohydrates. SCFAs serve as a source of energy for intestinal cells, a histone deacetylase inhibitor that regulates immune homeostasis, and/or a mediator of neurotransmitter production [65]. The observed negative correlations between Lachnospiraceae and Ruminococcaceae abundances with depressive symptoms in our study were consistent with previous studies [51, 66]. Park et al. [65] proposed that a reduction in the abundances of fermentationrelated bacteria may suggest a decay in SCFA production, which in turn may be associated with intestinal barrier dysfunction and an inflammatory response that can cause depression [67]. In another study, decreased levels of butyrateproducing bacteria (Ruminococcaceae_UG-013 and Lacknospiraceae_NK4A136_group) were found to be associated with high levels of inflammatory factors such as CRP and TNFalpha [68]. There is also clinical evidence of a positive association between stool acetate levels with depressive symptoms, as well as negative associations between butyrate and propionate levels with depressive symptoms [69]. While the associations between gut bacterial abundances, SCFA levels, gut physiology, and depressive symptoms require further study, SCFA levels have been proposed as potential noninvasive biomarkers for various health conditions [70] including depression [71]. For instance, there is clinical evidence of improvement of depression and an increase in the SCFAproducing bacteria (e.g., Roseburia, Ruminococcus, and Eubacterium) with an almond-based low carbohydrate diet [72] in patients with chronic diseases. Additionally, a recent review on correlations between gut bacterial metabolites with depression suggests that gut bacteria (i.e., Escherichia spp and Lactobacillus plantarum) may be a new target for depression because of their ability to regulate the neurotransmitter seroto$\operatorname{nin}(5-\mathrm{HT})$ levels in the body [71]. This is important because a disrupted serotonergic system may lead to depressive symptoms [71]. As such, besides bacterial abundances, SCFAs may be useful as potential biomarkers for the assessment of depression [71] and as potential therapeutic antiinflammatory agents [73, 74] for depression [75].
The positive correlation of Lactobacillus abundance and negative correlation of Phascolarctobacterium, Intestimonas/Megamonas, and Parasutterella abundances with depressive symptom scores in our study differed from findings from previous research. Lower Lactobacillus abundances were previously detected in chronic variable stress-induced depression rat model compared to controls using 16S rRNA gene sequencing [76]. Higher Phascolarctobacterium, Intestimonas/Megamonas, and Parasutterella abundances were previously detected in active-MDD patients compared to controls using reverse transcriptionquantitative polymerase chain reaction and 16S rRNA gene pyrosequencing approaches [51]. Nevertheless, besides depression, Streptococcus and Parasutterella abundances may also be affected by rectal cancer itself or by treatment side effects $[17,73,77]$. For example, a combination of fecal bacterial candidates, including Streptococcus bovis/Streptococcus gallolyticus, was proposed as biomarkers for early detection of adenomatous polyps and colon cancer [73, 77]. Further, increased abundance of Parasutterella was related to chemotherapy-induced gastrointestinal toxicity in animal models [78]. It is, of course, possible that our study findings differed from findings in previous research because most of the available literature is based on the clinical diagnostic classification for depression while our study focuses on depressive symptoms.

Our investigation revealed the finding that our multiple regression model explained $28 \%$ of the observed variance with $p<0.001$ and suggested that patients with higher depression scores tend to have higher levels of Bacillales and lower levels of Ruminococcaceae. Future larger-scale studies using animal models and/or anaerobic culture will be useful in confirming the functional roles of these specific taxa in depression and their utility as microbial biomarkers for depression [11]. While for our sample, bacterial genera were significantly associated with depressive symptom scores; it is important to acknowledge that depressed mood is a multifactorial condition influenced by multiple contributing factors (e.g., tumor type, prior psychological factors (preexisting mental health problems and their severity), psychological response to diagnoses (coping behavior), and social and contextual factors (unemployment and lack of social support); [79]. However, findings of our study suggest that gut microbiome disruption may be present before overt clinical depression is recognized, a finding that may prove useful in future research aimed at identifying biomarkers to aid in the establishment of clinical depression. Associations between other relevant factors including social and contextual factors potentially contributing to depressive symptoms among people with cancer have been supported in prior exhaustive reviews [79]. Further research that includes biological factors, as well as behavioral factors such as lack of coping strategy for handling the trauma of a cancer diagnosis, is required. This knowledge may shed light on personalized medicine/interventions for risk factor reduction.

As previously stated, the underpinnings of CRT-related depressive symptoms have not been fully elucidated. Based on the MetaCyc pathway predictions [80], depressive symptoms appeared to correlate mainly with biosynthetic 
pathways (e.g., flavin biosynthesis and S-adenosyl-L-methionine biosynthesis). This is remarkable since our pathway predictions point to mechanisms that researchers have proposed to contribute to the biologic underpinnings of depression: gut dysbiosis resulting in low production of micronutrients and gut dysbiosis promotion of inflammation $[11,80]$. In fact, riboflavin (vitamin B2) deficiency has been associated with depression in women [81], and the flavin biosynthesis I pathway was inversely associated with depressive symptoms in our study. Acetyl CoA fermentation to butanoate II was also inversely associated with depressive symptoms. This is interesting because there is evidence that acetyl-CoA is one of the pathways known for the production of the anti-inflammatory SCFA butyrate [17, 80, 82]. These findings also have further research implications since riboflavin supplementation has been suggested to improve mood in individuals [83], and sodium butyrate administration was reported to have antidepressant-like effects in animal models [84]. However, others have found in animal models that administration of the sodium propionate (also a SCFA) but not sodium butyrate induces antidepressant-like effects [85]. Further, the finding that the S-adenosyl methionine biosynthesis pathway was negatively correlated with depressive symptom scores is interesting since S-adenosyl methionine has been used as an antidepression supplement [86]. Two degradation pathways (chondroitin sulfate and myo-, chiro-, and scyllo-inositol) were also negatively correlated with depressive symptom scores. Chondroitin sulfate has been found to play an important role in synaptic transmission and plasticity in animal models [87] while evidence suggests that inositol may play a role in the management/ alleviation of depression [88]. Conversely, the positive correlation of mono-trans, poly-cis decaprenyl phosphate biosynthetic pathway abundances with depressive symptom scores may be linked to similar increases in Actinobacteria (such as Actinomyces) abundances with depressive symptom scores, since this pathway is found in Mycobacterium species classified to the phylum Actinobacteria $[89,90]$. Therefore, these candidate pathways and their metabolites warrant further investigation as they may shed light on the assessment and treatment of CRT-related symptoms such as depression and fatigue [14].

\section{Limitations}

Our results must be interpreted with caution as our pilot study has notable limitations including the brief duration of the follow-up and lack of a control group. For example, we cannot rule out that the influence of CRT on depressive symptoms could be due to the cancer nor can we rule out the confounding effects of cancer and CRT on the gut microbiome composition. Inclusion of a control group of patients without CRT could have strengthened the inference on the influence of CRT on depressive symptoms, as would the collection of stool samples before CRT to assess microbiota changes impacted by the CRT administration and controlling for CRT side effects. Additional studies with larger numbers of participants are needed to further examine the role of other factors (e.g., tumor samples, lifestyle, social environmental factors, pain, stress, diet, and diarrhea) that may affect the variability of the gut microbiota measurements and/or depressive symptoms in patients with rectal cancer. As the outcomes of this study were dependent on clinician-rated depressive symptoms, future research may also consider comparing our findings with participants' self-reported depressive symptoms perhaps using other validated measures of depression (e.g., the PROMIS depression forms). The availability of a larger sample size would permit the exclusion of somatic symptoms of depression in future studies. Controlling for these and other behavioral manifestations of depressive symptoms may inform the impact of these symptoms on gut microbiota and address a needed gap in the literature.

\section{Conclusions}

Overall, our results indicated that rectal cancer patients experience increases in depressive symptoms before CRT, which can worsen after treatment. We also identified taxa and predicted pathways that were significantly correlated with depressive symptom scores. Our findings served to inform future larger-scale clinical studies involving not only genuslevel 16S rRNA gene analysis but also metatranscriptomics, metagenomics, culturing experiments, and animal models. These future studies will contribute to the body of the literature of the underpinnings of depressive symptoms and clinical depression and facilitate the identification of microbial targets for effective diagnosis and clinical interventions.

\section{Data Availability}

The data used to support the findings of this study are available from the corresponding author upon request. It will be deposited in a database when the study is complete.

\section{Disclosure}

The content is solely the responsibility of the authors and does not necessarily represent the official views of the NIH.

\section{Conflicts of Interest}

The authors declare that they have no conflicts of interest.

\section{Acknowledgments}

We thank all the staff at Tampa General Hospital Cancer Center, the Cancer Care team at AdventHealth Tampa, and the St. Joseph's Hospital Cancer Institute for the collaborative clinical recruitment support. This study would not have been possible without the participants. We also thank the Biobehavioral Laboratory at the University of South Florida's College of Nursing for Illumina sequencing support. Thanks are extended to Miss Sara Marrero for her insightful proofreading and editing role. This work was supported by the National Institute of Nursing Research (NINR) of the National Institutes of Health (NIH) under Award number K23NR020039. 


\section{References}

[1] R. L. Siegel, K. D. Miller, and A. Jemal, "Cancer statistics, 2019,” CA: a Cancer Journal for Clinicians, vol. 69, no. 1, pp. 7-34, 2019.

[2] V. J. González-Mercado, W. A. Henderson, A. Sarkar et al., "Changes in gut microbiome associated with co-occurring symptoms development during chemo-radiation for rectal cancer: a proof of concept study," Biological Research for Nursing, vol. 23, no. 1, pp. 31-41, 2021.

[3] National Comprehensive Cancer Network, "Clinical practice guidelines in oncology; rectal cancer," 2016, https://www .nccn.org/Store/Login/Register.aspx? returnurl=https\%3a\% $2 \mathrm{f} \% 2 \mathrm{fwww}$.nccn.org\%2fprofessionals $\% 2 \mathrm{fphysician \_ gls} \%$ $2 \mathrm{fpdf} \% 2$ frectal.pdf.

[4] Y. Chongpison, M. C. Hornbrook, R. B. Harris et al., "Selfreported depression and perceived financial burden among long-term rectal cancer survivors," Psychooncology, vol. 25, no. 11, pp. 1350-1356, 2016.

[5] T. K. Gosselin, S. Beck, D. H. Abbott et al., "The symptom experience in rectal cancer survivors," Journal of Pain and Symptom Management, vol. 52, no. 5, pp. 709-718, 2016.

[6] L. J. F. Santos, J. B. S. Garcia, J. S. Pacheco, É. B. M. Vieira, and A. M. Santos, "Quality of life, pain, anxiety and depression in patients surgically treated with cancer of rectum," Arquivos Brasileiros de Cirurgia Digestiva, vol. 27, no. 2, pp. 96-100, 2014.

[7] T. McCarter, "Depression overview," American Health \& Drug Benefits, vol. 1, no. 3, pp. 44-51, 2008.

[8] E. H. Shinn, A. Valentine, A. Jethanandani et al., "Depression and oropharynx cancer outcome," Psychosomatic Medicine, vol. 78, no. 1, pp. 38-48, 2016.

[9] T. L. Huang and C. C. Lin, "Advances in biomarkers of major depressive disorder," Advances in Clinical Chemistry, vol. 68, pp. 177-204, 2015.

[10] T. C. Fung, C. A. Olson, and E. Y. Hsiao, "Interactions between the microbiota, immune and nervous systems in health and disease," Nature Neuroscience, vol. 20, no. 2, pp. 145-155, 2017.

[11] S. G. Cheung, A. R. Goldenthal, A. C. Uhlemann, J. J. Mann, J. M. Miller, and M. E. Sublette, "Systematic review of gut microbiota and major depression," Frontiers in Psychiatry, vol. 10, p. 34, 2019.

[12] K. Sanada, S. Nakajima, S. Kurokawa et al., "Gut microbiota and major depressive disorder: a systematic review and metaanalysis," Journal of Affective Disorders, vol. 266, pp. 1-13, 2020.

[13] N. B. Perez, C. Dorsen, and A. Squires, "Dysbiosis of the gut microbiome: a concept analysis," Journal of Holistic Nursing, vol. 38, no. 2, pp. 223-232, 2020.

[14] V. J. González-Mercado, J. Lim, S. Marrero, E. Pedro, and L. N. Saligan, "Gut microbiota and fatigue in rectal cancer patients: a cross-sectional pilot study," Supportive Care in Cancer, vol. 29, no. 8, pp. 4615-4621, 2021.

[15] V. J. Gonzalez-Mercado, J. Perez-Santiago, D. Lyon et al., “The role of gut microbiome perturbation in fatigue induced by repeated stress from chemoradiotherapy: a proof of concept study," Advances in Medicine, vol. 2020, Article ID 6375876, 2020.

[16] V. J. González-Mercado, A. Sarkar, F. J. Penedo et al., “Gut microbiota perturbation is associated with acute sleep distur- bance among rectal cancer patients," Journal of Sleep Research, vol. 29, no. 3, p. e12915, 2020.

[17] V. J. González-Mercado, J. Lim, G. Yu et al., "Co-occurrence of symptoms and gut microbiota composition before neoadjuvant chemotherapy and radiation therapy for rectal cancer: a proof of concept," Biological Research for Nursing, vol. 23, no. 3, pp. 513-523, 2021.

[18] V. J. González-Mercado, J. Lim, L. Berk, M. Esele, C. S. Rodríguez, and G. Colón-Otero, "Gut microbiota differences in Island Hispanic Puerto Ricans and mainland non- Hispanic whites during chemoradiation for rectal cancer: a pilot study," Current Problems in Cancer, vol. 44, no. 4, article 100551, 2020.

[19] M. Hamilton, “A rating scale for depression," Journal of Neurology, Neurosurgery, and Psychiatry, vol. 23, no. 1, pp. 5662, 1960.

[20] J. B. Williams, "A structured interview guide for the Hamilton Depression Rating Scale," Archives of General Psychiatry, vol. 45 , no. 8 , pp. $742-747,1988$.

[21] A. Kieslich da Silva, M. Reche, A. Lima, M. P. A. Fleck, E. Capp, and F. M. Shansis, "Assessment of the psychometric properties of the 17- and 6-item Hamilton Depression Rating Scales in major depressive disorder, bipolar depression and bipolar depression with mixed features," Journal of Psychiatric Research, vol. 108, pp. 84-89, 2019.

[22] W. M. Lydiatt, D. Denman, D. P. McNeilly, S. E. Puumula, and W. J. Burke, "A randomized, placebo-controlled trial of citalopram for the prevention of major depression during treatment for head and neck cancer," Archives of Otolaryngology - Head \& Neck Surgery, vol. 134, no. 5, pp. 528-535, 2008.

[23] R. Caspi, R. Billington, I. M. Keseler et al., "The MetaCyc database of metabolic pathways and enzymes - a 2019 update," Nucleic Acids Research, vol. 48, no. D1, pp. D445-D453, 2020.

[24] M. G. Langille, J. Zaneveld, J. G. Caporaso et al., "Predictive functional profiling of microbial communities using $16 \mathrm{~S}$ rRNA marker gene sequences," Nature Biotechnology, vol. 31, no. 9, pp. 814-821, 2013.

[25] A. J. Ferrari, F. J. Charlson, R. E. Norman et al., "Burden of depressive disorders by country, sex, age, and year: findings from the global burden of disease study 2010," PLoS Medicine, vol. 10, no. 11, article e1001547, 2013.

[26] J. L. Sotelo, D. Musselman, and C. Nemeroff, "The biology of depression in cancer and the relationship between depression and cancer progression," International Review of Psychiatry, vol. 26, no. 1, pp. 16-30, 2014.

[27] R. M. Saracino and C. J. Nelson, "Identification and treatment of depressive disorders in older adults with cancer," Journal of Geriatric Oncology, vol. 10, no. 5, pp. 680-684, 2019.

[28] R. Bandu, H. J. Lee, H. M. Lee et al., "Liquid chromatography/ mass spectrometry-based plasma metabolic profiling study of escitalopram in subjects with major depressive disorder," Journal of Mass Spectrometry, vol. 53, no. 5, pp. 385-399, 2018.

[29] U. Polat, A. Arpaci, S. Demir, S. Erdal, and S. Yalcin, "Evaluation of quality of life and anxiety and depression levels in patients receiving chemotherapy for colorectal cancer: impact of patient education before treatment initiation," Journal of Gastrointestinal Oncology, vol. 5, no. 4, pp. 270-275, 2014.

[30] J. E. Bajic, I. N. Johnston, G. S. Howarth, and M. R. Hutchinson, "From the bottom-up: chemotherapy and gut-brain axis dysregulation," Frontiers in Behavioral Neuroscience, vol. 12, p. 104, 2018. 
[31] B. A. Hollingsworth, D. R. Cassatt, A. L. DiCarlo et al., "Acute radiation syndrome and the microbiome: impact and review," Frontiers in Pharmacology, vol. 12, article 643283, 2021.

[32] J. M. Deleemans, F. Chleilat, R. A. Reimer et al., "The chemo-gut study: investigating the long-term effects of chemotherapy on gut microbiota, metabolic, immune, psychological and cognitive parameters in young adult cancer survivors; study protocol," BMC Cancer, vol. 19, no. 1, p. 1243, 2019.

[33] K. R. Jordan, B. R. Loman, M. T. Bailey, and L. M. Pyter, "Gut microbiota-immune-brain interactions in chemotherapyassociated behavioral comorbidities," Cancer, vol. 124, no. 20, pp. 3990-3999, 2018.

[34] B. C. Song and J. Bai, "Microbiome-gut-brain axis in cancer treatment-related psychoneurological toxicities and symptoms: a systematic review," Supportive Care in Cancer, vol. 29, no. 2, pp. 605-617, 2021.

[35] R. Hills, B. Pontefract, H. Mishcon, C. Black, S. Sutton, and S. Theberge, "Gut microbiome: profound implications for diet and disease," Nutrients, vol. 11, no. 7, p. 1613, 2019.

[36] M. C. Flux and C. A. Lowry, "Finding intestinal fortitude: integrating the microbiome into a holistic view of depression mechanisms, treatment, and resilience," Neurobiology of Disease, vol. 135, article 104578, 2020.

[37] P. Zheng, B. Zeng, C. Zhou et al., "Gut microbiome remodeling induces depressive-like behaviors through a pathway mediated by the host's metabolism," Molecular Pychiatry, vol. 21, no. 6, pp. 786-796, 2016.

[38] M. Pirbaglou, J. Katz, R. J. de Souza, J. C. Stearns, M. Motamed, and P. Ritvo, "Probiotic supplementation can positively affect anxiety and depressive symptoms: a systematic review of randomized controlled trials," Nutrition Research, vol. 36, no. 9, pp. 889-898, 2016.

[39] C. Wallace and R. Milev, "The effects of probiotics on depressive symptoms in humans: a systematic review," Annals of General Psychiatry, vol. 16, no. 1, p. 14, 2017.

[40] R. D. Nipp, G. Fuchs, A. El-Jawahri et al., "Sarcopenia is associated with quality of life and depression in patients with advanced cancer," The Oncologist, vol. 23, no. 1, pp. 97-104, 2018.

[41] S. A. Pötgens, H. Brossel, M. Sboarina et al., “_Klebsiella oxytoca_expands in cancer cachexia and acts as a gut pathobiont contributing to intestinal dysfunction," Scientific Reports, vol. 8, no. 1, p. 12321, 2018.

[42] H. Sun, T. Sudip, X. Fu, S. Wen, H. Liu, and S. Yu, "Cachexia is associated with depression, anxiety and quality of life in cancer patients," BMJ Supportive \& Palliative Care, 2020.

[43] M. J. Tisdale, "Mechanisms of cancer cachexia," Physiological Reviews, vol. 89, no. 2, pp. 381-410, 2009.

[44] M. L. Chen and H. K. Chang, "Physical symptom profiles of depressed and nondepressed patients with cancer," Palliative Medicine, vol. 18, no. 8, pp. 712-718, 2004.

[45] B. Liu, X. Wu, L. Shi et al., "Correlations of social isolation and anxiety and depression symptoms among patients with breast cancer of Heilongjiang province in China: the mediating role of social support," Nursing Open, vol. 8, no. 4, pp. 19811989, 2021.

[46] F. Dunphy-Doherty, S. M. O'Mahony, V. L. Peterson et al., "Post-weaning social isolation of rats leads to long-term disruption of the gut microbiota-immune-brain axis," Brain, Behavior, and Immunity, vol. 68, pp. 261-273, 2018.
[47] L. Feng, W. Zhang, Q. Shen et al., "Bile acid metabolism dysregulation associates with cancer cachexia: roles of liver and gut microbiome," Journal of Cachexia, Sarcopenia and Muscle, 2021.

[48] K. M. Herremans, A. N. Riner, M. E. Cameron, and J. G. Trevino, "The microbiota and cancer cachexia," International Journal of Molecular Sciences, vol. 20, no. 24, p. 6267, 2019.

[49] E. Rinninella, P. Raoul, M. Cintoni et al., "What is the healthy gut microbiota composition? A changing ecosystem across age, environment, diet, and diseases," Microorganisms, vol. 7, no. 1, p. 14, 2019.

[50] Z. Chen, J. Li, S. Gui et al., "Comparative metaproteomics analysis shows altered fecal microbiota signatures in patients with major depressive disorder," Neuroreport, vol. 29, no. 5, pp. 417-425, 2018.

[51] H. Jiang, Z. Ling, Y. Zhang et al., "Altered fecal microbiota composition in patients with major depressive disorder," Brain, Behavior, and Immunity, vol. 48, pp. 186-194, 2015.

[52] P. Lin, B. Ding, C. Feng et al., "Prevotella and Klebsiella proportions in fecal microbial communities are potential characteristic parameters for patients with major depressive disorder," Journal of Affective Disorders, vol. 207, pp. 300304, 2017.

[53] T. Barichello, L. R. Simões, J. S. Generoso et al., "Depressionlike adult behaviors may be a long-term result of experimental pneumococcal meningitis in Wistar rats infants," Neurochemical Research, vol. 41, no. 10, pp. 2771-2778, 2016.

[54] K. Yaddanapudi, M. Hornig, R. Serge et al., "Passive transfer of streptococcus-induced antibodies reproduces behavioral disturbances in a mouse model of pediatric autoimmune neuropsychiatric disorders associated with streptococcal infection," Molecular Psychiatry, vol. 15, no. 7, pp. 712-726, 2010.

[55] J. E. Clarridge 3rd and Q. Zhang, "Genotypic diversity of clinical Actinomyces species: phenotype, source, and disease correlation among genospecies," Journal of Clinical Microbiology, vol. 40, no. 9, pp. 3442-3448, 2002.

[56] M. Gajdács and E. Urbán, “The Pathogenic Role of Actinomyces spp. and Related Organisms in Genitourinary Infections: Discoveries in the New, Modern Diagnostic Era," Antibiotics, vol. 9, no. 8, p. 524, 2020.

[57] V. Hinić, C. Straub, E. Schultheiss, P. Kaempfer, R. Frei, and D. Goldenberger, "Identification of a novel 16S rRNA gene variant of Actinomyces funkei from six patients with purulent infections," Clinical Microbiology and Infection, vol. 19, no. 7, pp. E312-E314, 2013.

[58] N. B. Perez, F. Wright, and A. Vorderstrasse, "A microbial relationship between irritable bowel syndrome and depressive symptoms," Biological Research for Nursing, vol. 23, no. 1, pp. 50-64, 2021.

[59] R. Pittayanon, J. T. Lau, G. I. Leontiadis et al., "Differences in gut microbiota in patients with vs without inflammatory bowel diseases: a systematic review," Gastroenterology, vol. 158, no. 4, pp. 930-946.e1, 2020.

[60] G. G. Nascimento, M. T. Gastal, F. Leite et al., "Is there an association between depression and periodontitis? A birth cohort study," Journal of Clinical Periodontology, vol. 46, no. 1, pp. 31-39, 2019.

[61] R. Neuendorf, A. Harding, N. Stello, D. Hanes, and H. Wahbeh, "Depression and anxiety in patients with inflammatory bowel disease: a systematic review," Journal of Psychosomatic Research, vol. 87, pp. 70-80, 2016. 
[62] T.-T. Huang, J.-B. Lai, Y. L. du, Y. Xu, L. M. Ruan, and S.-H. Hu, "Current understanding of gut microbiota in mood disorders: an update of human studies," Frontiers in Genetics, vol. 10, 2019.

[63] D. Macedo, A. Filho, C. N. Soares de Sousa et al., "Antidepressants, antimicrobials or both? Gut microbiota dysbiosis in depression and possible implications of the antimicrobial effects of antidepressant drugs for antidepressant effectiveness," Journal of Affective Disorders, vol. 208, pp. 22-32, 2017.

[64] P. J. W. Naude, S. Claassen-Weitz, S. Gardner-Lubbe et al., "Association of maternal prenatal psychological stressors and distress with maternal and early infant faecal bacterial profile," Acta Neuropsychiatrica, vol. 32, no. 1, pp. 32-42, 2020.

[65] M. Park, J. Choi, and H. J. Lee, "Flavonoid-rich orange juice intake and altered gut microbiome in young adults with depressive symptom: a randomized controlled study," Nutrients, vol. 12, no. 6, p. 1815, 2020.

[66] A. Naseribafrouei, K. Hestad, E. Avershina et al., "Correlation between the human fecal microbiota and depression," Neurogastroenterology and Motility, vol. 26, no. 8, pp. 1155-1162, 2014.

[67] A. L. Lopresti, G. L. Maker, S. D. Hood, and P. D. Drummond, "A review of peripheral biomarkers in major depression: the potential of inflammatory and oxidative stress biomarkers," Progress in Neuro-Psychopharmacology \& Biological Psychiatry, vol. 48, pp. 102-111, 2014.

[68] M. Duan, F. Liu, H. Fu, S. Lu, and T. Wang, "Preoperative microbiomes and intestinal barrier function can differentiate prodromal Alzheimer's disease from normal neurocognition in elderly patients scheduled to undergo orthopedic surgery," Frontiers in Cellular and Infection Microbiology, vol. 11, article 592842, 2021.

[69] B. Müller, A. J. Rasmusson, D. Just et al., "Fecal short-chain fatty acid ratios as related to gastrointestinal and depressive symptoms in young adults," Psychosomatic Medicine, vol. 83, no. 7, pp. 693-699, 2021.

[70] T. Pang, S. T. Leach, T. Katz, A. S. Day, and C. Y. Ooi, "Fecal biomarkers of intestinal health and disease in children," Frontiers in Pediatrics, vol. 2, p. 6, 2014.

[71] O. V. Averina, Y. A. Zorkina, R. A. Yunes et al., "Bacterial metabolites of human gut microbiota correlating with depression," International Journal of Molecular Sciences, vol. 21, no. 23, p. 9234, 2020.

[72] M. Ren, H. Zhang, J. Qi et al., “An almond-based low carbohydrate diet improves depression and glycometabolism in patients with type 2 diabetes through modulating gut microbiota and GLP-1: a randomized controlled trial," Nutrients, vol. 12, no. 10, p. 3036, 2020.

[73] J. Butt, S. Werner, M. Willhauck-Fleckenstein et al., "Serology ofStreptococcus gallolyticussubspeciesgallolyticusand its association with colorectal cancer and precursors," International Journal of Cancer, vol. 141, no. 5, pp. 897-904, 2017.

[74] C. Nastasi, M. Candela, C. M. Bonefeld et al., "The effect of short-chain fatty acids on human monocyte-derived dendritic cells," Scientific Reports, vol. 5, no. 1, article 16148, 2015.

[75] G. Caspani, S. Kennedy, J. A. Foster, and J. Swann, "Gut microbial metabolites in depression: understanding the biochemical mechanisms," Microbial Cell, vol. 6, no. 10, pp. 454-481, 2019.

[76] M. Yu, H. Jia, C. Zhou et al., "Variations in gut microbiota and fecal metabolic phenotype associated with depression by $16 \mathrm{~S}$ rRNA gene sequencing and LC/MS-based metabolomics,"
Journal of Pharmaceutical and Biomedical Analysis, vol. 138, pp. 231-239, 2017.

[77] S. Rezasoltani, M. Sharafkhah, H. Asadzadeh Aghdaei et al., "Applying simple linear combination, multiple logistic and factor analysis methods for candidate fecal bacteria as novel biomarkers for early detection of adenomatous polyps and colon cancer," Journal of Microbiological Methods, vol. 155, pp. 82-88, 2018.

[78] R. A. Forsgård, V. G. Marrachelli, K. Korpela et al., "Chemotherapy-induced gastrointestinal toxicity is associated with changes in serum and urine metabolome and fecal microbiota in male Sprague-Dawley rats," Cancer Chemotherapy and Pharmacology, vol. 80, no. 2, pp. 317-332, 2017.

[79] C. L. Niedzwiedz, L. Knifton, K. A. Robb, S. V. Katikireddi, and D. J. Smith, "Depression and anxiety among people living with and beyond cancer: a growing clinical and research priority," BMC Cancer, vol. 19, no. 1, p. 943, 2019.

[80] R. T. Liu, A. D. Rowan-Nash, A. E. Sheehan et al., "Reductions in anti-inflammatory gut bacteria are associated with depression in a sample of young adults," Brain, Behavior, and Immunity, vol. 88, pp. 308-324, 2020.

[81] M. Naghashpour, R. Amani, R. Nutr, S. Nematpour, and M. H. Haghighizadeh, "Riboflavin status and its association with serum hs-CRP levels among clinical nurses with depression," Journal of the American College of Nutrition, vol. 30, no. 5, pp. 340-347, 2011.

[82] M. Vital, A. C. Howe, and J. M. Tiedje, "Revealing the bacterial butyrate synthesis pathways by analyzing (meta) genomic data," mBio, vol. 5, no. 2, article e00889, 2014.

[83] T. S. Rao, M. R. Asha, B. N. Ramesh, and K. S. Rao, "Understanding nutrition, depression and mental illnesses," Indian Journal of Psychiatry, vol. 50, no. 2, pp. 77-82, 2008.

[84] W. R. Resende, S. S. Valvassori, G. Z. Réus et al., "Effects of sodium butyrate in animal models of mania and depression: implications as a new mood stabilizer," Behavioural Pharmacology, vol. 24, no. 7, pp. 569-579, 2013.

[85] J. Li, L. Hou, C. Wang, X. Jia, X. Qin, and C. Wu, "Short term intrarectal administration of sodium propionate induces antidepressant-like effects in rats exposed to chronic unpredictable mild stress," Frontiers in Psychiatry, vol. 9, p. 454, 2018.

[86] I. Galizia, L. Oldani, K. Macritchie et al., "S-Adenosyl methionine (SAMe) for depression in adults," Cochrane Database of Systematic Reviews, vol. 10, no. 10, article CD011286, 2016.

[87] E. Albiñana, J. Gutierrez-Luengo, N. Hernández-Juarez et al., "Chondroitin sulfate induces depression of synaptic transmission and modulation of neuronal plasticity in rat hippocampal slices," Neural Plasticity, vol. 2015, Article ID 463854, 2015.

[88] T. Mukai, T. Kishi, Y. Matsuda, and N. Iwata, "A meta-analysis of inositol for depression and anxiety disorders," Human Psychopharmacology, vol. 29, no. 1, pp. 55-63, 2014.

[89] D. C. Crick, M. C. Schulbach, E. E. Zink et al., "Polyprenyl phosphate biosynthesis in Mycobacterium tuberculosis and Mycobacterium smegmatis," Journal of Bacteriology, vol. 182, no. 20 , pp. $5771-5778,2000$.

[90] D. Kaur, P. J. Brennan, and D. C. Crick, "Decaprenyl diphosphate synthesis in Mycobacterium tuberculosis," Journal of Bacteriology, vol. 186, no. 22, pp. 7564-7570, 2004. 